[2] N. E. Leonard and E. Fiorelli, "Virtual leaders, artificial potentials and coordinated control of groups," in Proc. IEEE Conf. Decision Control, Orlando, FL, 2001, pp. 2968-2973.

[3] R. T. Jonathan, R. W. Beard, and B. J. Young, "A decentralized approach to formation maneuvers," IEEE Trans. Robot. Automat., vol. 19, no. 6, pp. 933-941, Dec, 2003.

[4] T. Balch and R. C. Arkin, "Behavior-based formation control for multirobot teams," IEEE Trans. Robot. Automat., vol. 14, no. 6, pp. 926-939, Dec. 1998.

[5] M. A. Lewis and K.-H. Tan, "High precision formation control of mobile robots using virtual structures," Auton. Robot., vol. 4, pp. 387-403, 1997.

[6] R. Skjetne, S. Moi, and T. I. Fossen, "Nonlinear formation control of marine craft," in Proc. IEEE Conf. Decision Control, Las Vegas, NV, 2002, pp. 1699-1704.

[7] D. M. Stipanovica, G. Inalhana, R. Teo, and C. J. Tomlina, "Decentralized overlapping control of a formation of unmanned aerial vehicles," Automatica, vol. 40, pp. 1285-1296, 2004.

[8] H. G. Tanner and A. Kumar, "Towards decentralization of multi-robot navigation functions," in Proc. IEEE Int. Conf. Robot. Automat., Barcelona, Spain, 2005, pp. 4143-4148.

[9] — " "Formation stabilization of multiple agents using decentralized navigation functions," in Robotics: Science and Systems IS. Thrun, G. Sukhatme, S. Schaal, and O. Brock, Eds. Cambridge, MA: MIT Press, 2005, p. 4956.

[10] E. Rimon and D. E. Koditschek, "Robot navigation functions on manifolds with boundary," Adv. Appl. Math., vol. 11, pp. 412-442, 1990.

[11] V. Gazi and K. M. Passino, "A class of attraction/repulsion functions for stable swarm aggregations," Int. J. Control, vol. 77, pp. 1567-1579, 2004.

[12] H. G. Tanner, A. Jadbabaie, and G. J. Pappas, "Stable flocking of mobile agents-Part II: Dynamics topology," in Proc. IEEE Conf. Decision Control, Honolulu, HI, 2003, pp. 2016-2021.

[13] S. S. Ge and Y. J. Cui, "New potential functions for mobile robot path planning," IEEE Trans. Robot. Automat., vol. 16, no. 5, pp. 615-620, Oct. 2000.

[14] P. Ogren, M. Egerstedt, and X. Hu, "A control Lyapunov function approach to multi-agent coordination," IEEE Trans. Robot. Automat., vol. 18 , no. 5, pp. 847-851, Oct. 2002.

[15] P. Ogren, E. Fiorelli, and N. E. Leonard, "Cooperative control of mobile sensor networks: Adaptive gradient climbing in a distributed environment," IEEE Trans. Autom. Control, vol. 49, no. 8, pp. 1292-1302, Aug. 2004.

[16] J. Cortés, S. Martínez, T. Karatas, and F. Bullo, "Coverage control for mobile sensing networks," IEEE Trans. Robot. Automat., vol. 20, no. 2, pp. 243-255, Mar. 2004.

[17] H. Khalil, Nonlinear Systems. Upper Saddle River, NJ: Prentice-Hall, 2002.

[18] A. Wells, Theory and Problems of Lagrangian Dynamics. New York: Springer-Verlag, 1967.

[19] K. D. Do, Z. P. Jiang, and J. Pan, "Universal controllers for stabilization and tracking of underactuated ships," Syst. Control Lett., vol. 47, pp. 299-317, 2002.

\section{Piecewise Linear Identification for the Rate-Independent and Rate-Dependent Duhem Hysteresis Models}

\author{
JinHyoung Oh and Dennis S. Bernstein, Fellow, IEEE
}

Abstract-We consider the semilinear Duhem model and develop an identification method for rate-independent and rate-dependent hysteresis. For rate-independent hysteresis, we reparameterize the system in terms of the input signal, so that the system has the form of a switching linear time-invariant system with ramp-plus-step forcing. For rate-dependent hysteresis, the system can be viewed as a switching linear time-invariant system for triangle wave inputs. Least-squares-based methods are developed to identify the rate-independent and rate-dependent semilinear Duhem models.

Index Terms-Duhem model, hysteresis, rate dependence.

\section{INTRODUCTION}

Hysteresis is a nonlinear phenomenon that arises in a wide range of disciplines. Hysteretic systems have the special property that the input-output closed curve remains nontrivial (possesses interior points) as the frequency content of the input signal approaches dc. A nonlinear system is hysteretic if it possesses a nontrivial quasi-dc input-output closed curve. If the input-output closed curve is independent of the time-scaling of the input signal, the hysteretic system is rate independent. If the input-output closed curve is input frequency dependent, and thus the hysteretic response near dc is different from the input-output response at higher frequencies, the system is rate dependent. More detail on these definitions is given in [1].

In this note, we develop identification methods for rate-independent and rate-dependent hysteresis in the Duhem model [1]-[3]. While the literature contains a wide variety of hysteresis models (see [4] and the references therein), the Duhem model is a nonlinear ordinary differential equation that can model either rate-independent or rate-dependent hysteresis. The Duhem model is widely used for friction modeling, and includes the Dahl, LuGre, and Maxwell-slip models as special cases [5].

To estimate system parameters, we transform the identification problem into a piecewise-linear estimation problem. For rate-independent hysteresis, the system is reparameterized in terms of the input signal rather than time [1]. With this reparameterization, the semilinear Duhem model has the form of a switching linear system with ramp-plus-step forcing. For rate-dependent hysteresis, we use a special class of input signals, specifically, triangle waves, under which the system has the form of a switching linear system with ramp forcing.

After the transformations, the models are identified as piecewiseaffine ARX (PWARX) systems. The PWARX models that we identify in the present note are based on fixed switching guidelines, and thus standard least squares techniques can be used. The dynamics of the rate-dependent semilinear Duhem model are identified in a nonparametric, that is, pointwise, fashion.
Manuscript received November 15, 2005; revised September 23, 2006. Recommended by Associate Editor W. X. Zheng. This work was supported in part by the National Science Foundation under Grant ECS-0225799.

J. Oh is with Autoliv Electronics America, Southfield, MI 48034 USA (e-mail: jinhyoung.oh@autoliv.com).

D. S. Bernstein is with the Department of Aerospace Engineering, The University of Michigan, Ann Arbor, MI 48109-2140 USA (e-mail: dsbaero@umich. edu). 
Throughout the note, we use the terms closed curve, limiting periodic input-output map, hysteresis map, and rate-independence, which are defined in [1].

\section{RATE-INDEPENDENT SEMILINEAR DUHEM MODEL}

Consider the single-input-single-output (SISO) rate-independent semilinear Duhem model

$$
\begin{aligned}
\dot{x}(t)= & {\left[\begin{array}{ll}
\dot{u}_{+}(t) I_{n} & \dot{u}_{-}(t) I_{n}
\end{array}\right] } \\
& \times\left(\left[\begin{array}{l}
A_{+} \\
A_{-}
\end{array}\right] x(t)+\left[\begin{array}{l}
B_{+} \\
B_{-}
\end{array}\right] u(t)+\left[\begin{array}{l}
E_{+} \\
E_{-}
\end{array}\right]\right) \\
y(t)= & C x(t)+D u(t), \quad x(0)=x_{0}, \quad t \geq 0
\end{aligned}
$$

where $x:[0, \infty) \rightarrow \mathbb{R}^{n}$ is absolutely continuous, $u:[0, \infty) \rightarrow \mathbb{R}$ is continuous and piecewise $\mathrm{C}^{1}, A_{+} \in \mathbb{R}^{n \times n}, A_{-} \in \mathbb{R}^{n \times n}, B_{+} \in \mathbb{R}^{n}$, $B_{-} \in \mathbb{R}^{n}, E_{+} \in \mathbb{R}^{n}, E_{-} \in \mathbb{R}^{n}, C \in \mathbb{R}^{1 \times n}, D \in \mathbb{R}$, and $\dot{u}_{+}(t) \triangleq$ $\max \{0, \dot{u}(t)\}, \dot{u}_{-}(t) \triangleq \min \{0, \dot{u}(t)\}$. The value of $\dot{x}(t)$ at a point $t$ at which $\dot{u}(t)$ is discontinuous can be assigned arbitrarily. We assume that the solution to (1) exists and is unique on all finite intervals. Under these assumptions, $x$ and $y$ are continuous and piecewise $\mathrm{C}^{1}$. The following result from [1] is needed for further discussion.

Proposition 1: Consider the rate-independent semilinear Duhem model (1), (2). Let $\hat{x}:\left[u_{\min }, u_{\max }\right] \rightarrow \mathbb{R}^{n}$ and $\hat{y}:\left[u_{\min }, u_{\max }\right] \rightarrow \mathbb{R}$ satisfy

$$
\begin{aligned}
& \frac{\mathrm{d} \hat{x}(u)}{\mathrm{d} u}= \begin{cases}A_{+} \hat{x}(u)+B_{+} u+E_{+}, & \text {when } u \text { increases } \\
A_{-} \hat{x}(u)+B_{-} u+E_{-}, & \text {when } u \text { decreases } \\
0, & \text { otherwise }\end{cases} \\
& \hat{y}(u)=C \hat{x}(u)+D u
\end{aligned}
$$

for $u \in\left[u_{\min }, u_{\max }\right]$ and with initial condition $\hat{x}\left(u_{0}\right)=x_{0}$, where $u_{0} \in\left[u_{\min }, u_{\max }\right]$. Furthermore, let $u:[0, \infty) \rightarrow\left[u_{\min }, u_{\max }\right]$ be piecewise monotonic, continuous, piecewise $\mathrm{C}^{1}$, and $u(0)=u_{0}$. Then, $x(t) \triangleq \hat{x}(u(t))$ and $y(t) \triangleq \hat{y}(u(t))$ satisfy (1), (2).

Proposition 1 shows that the rate-independent semilinear Duhem model (1), (2) can be reparameterized with $u$ as the independent variable instead of $t$. This reparameterization transforms the time-domain model (1), (2) into a switching linear time-invariant system with rampplus-step inputs $B_{+} u+E_{-}$and $B_{-} u+E-$. Therefore, the input in (3) can be treated as a ramp-plus-step without consideration of the actual form of the time-domain input $u(t)$.

Let $\rho(A)$ denote the spectral radius of $A \in \mathbb{R}^{n \times n}$. The following result given in [1] provides a sufficient condition for the existence of a limiting periodic input-output map for a rate-independent semilinear Duhem model.

Theorem 1: Consider the rate-independent semilinear Duhem model (1), (2), where $u:[0, \infty) \rightarrow\left[u_{\min }, u_{\max }\right]$ is continuous, piecewise $\mathrm{C}^{1}$, and periodic with period $\alpha$ and has exactly one local maximum $u_{\max }$ in $[0, \alpha)$ and exactly one local minimum $u_{\min }$ in $[0, \alpha)$. Furthermore, define $\beta \triangleq u_{\max }-u_{\min }$ and assume that

$$
\rho\left(e^{\beta A_{+}} e^{-\beta A_{-}}\right)<1 .
$$

Then, (1) has a unique periodic solution $x:[0, \infty) \rightarrow \mathbb{R}^{n}$, and the limiting periodic input-output map $\mathcal{H}_{\infty}(u)$ exists.

\section{IDENTIFICATION OF THE RATE-INDEPENDENT SEMILINEAR DUHEM MODEL}

We now develop an identification method for systems with rate-independent hysteresis based on the rate-independent semilinear Duhem model (1), (2). Specifically, let a rate-independent hysteretic limiting periodic input-output map $\mathcal{H}_{\infty}(u)$ be given, where $u:[0, \infty) \rightarrow\left[u_{\min }, u_{\max }\right]$ is continuous, piecewise $\mathrm{C}^{1}$, and periodic with period $\alpha$ and have exactly one local maximum $u_{\max }$ in $[0, \alpha)$ and exactly one local minimum $u_{\min }$ in $[0, \alpha)$. Let $\hat{y}_{+}(u)$ be the subset of $\mathcal{H}_{\infty}(u)$ for which $u$ is increasing, and let $y_{-}(u)$ be the subset of $\mathcal{H}_{\infty}(u)$ for which $u$ is decreasing, such that $\mathcal{H}_{\infty}(u)=$ $\left\{\left(u, \hat{y}_{+}(u)\right): u \in\left[u_{\min }, u_{\max }\right]\right\} \cup\left\{\left(u, \hat{y}_{-}(u)\right): u \in\left[u_{\min }, u_{\max }\right]\right\}$. Then the rate-independent semilinear Duhem model identification problem is to find an order $n$ and matrices $A_{+} \in \mathbb{R}^{n \times n}, A_{-} \in \mathbb{R}^{n \times n}$, $B_{+} \in \mathbb{R}^{n}, B_{-} \in \mathbb{R}^{n}$, and $C \in \mathbb{R}^{1 \times n}$ such that the limiting periodic input-output map of (1), (2) coincides with $\mathcal{H}_{\infty}(u)$. Furthermore, to guarantee convergence to the hysteresis map, we require that the convergence condition $\rho\left(e^{\beta A_{1}}+e^{-\beta A}-\right)<1$ in Theorem 1 be satisfied. For convenience, we set $E_{+}=E_{-}=0$ and $D=0$.

Since the system to be identified is rate independent, we can reparameterize the identified semilinear Duhem model in terms of $u$ to obtain

$$
\begin{aligned}
\frac{\mathrm{d} \hat{x}(u)}{\mathrm{d} u} & = \begin{cases}A_{+} \hat{x}(u)+B_{+} u, & \text { when } u \text { increases } \\
A_{-} \hat{x}(u)+B_{-} u, & \text { when } u \text { decreases } \\
0, & \text { otherwise }\end{cases} \\
\hat{y}(u) & =C \hat{x}(u) .
\end{aligned}
$$

Then, the rate-independent semilinear Duhem model identification problem is equivalent to identifying two linear systems whose positive ramp response $B_{+} u$ in forward time and negative ramp response $B_{-} u$ in backward time coincide with $\hat{y}_{+}(u)$ and $\hat{y}_{-}(u)$, respectively, under the convergence condition (5).

Since the independent variable of the linear system (6) is nonmonotonic, we introduce the monotonically increasing independent variable $\hat{u} \in\left[u_{\min }, 2 u_{\max }-u_{\min }\right]$ to avoid backward-in-time identification. Then, we define a combined ramp input $\bar{u}$ in terms of the new independent variable $\hat{u}$ as

$$
\bar{u}(\hat{u}) \triangleq \begin{cases}\hat{u}, & u_{\min } \leq \hat{u}<u_{\max } \\ u_{\max }+u_{\min }-\hat{u}, & u_{\max } \leq \hat{u} \leq 2 u_{\max }-u_{\min } .\end{cases}
$$

Next, we reparameterize $\hat{y}_{+}(u)$ and $\hat{y}_{-}(u)$ in terms of $\hat{u}$ by "flipping over" $\hat{y}_{-}(u)$ and concatenating it to $\hat{y}_{+}(u)$. Specifically, define

$$
\bar{y}(\hat{u}) \triangleq \begin{cases}\hat{y}_{+}(\hat{u}), & u_{\min } \leq \hat{u}<u_{\max } \\ \hat{y}_{-}\left(u_{\max }+u_{\min }-\hat{u}\right), & u_{\max } \leq \hat{u} \leq 2 u_{\max }-u_{\min }\end{cases}
$$

Hence, the rate-independent semilinear Duhem model identification problem is equivalent to identifying a switching linear system with an input $\bar{u}(\hat{u})$ and output $\bar{y}(\hat{u})$ for $\hat{u} \in\left[u_{\min }, 2 u_{\max }-u_{\min }\right]$, where switching occurs at $\hat{u}=u_{\max }$ and subject to the convergence condition (5).

Now, let $u_{k}$ and $y_{k}, k=0, \ldots, 2 \ell-1$, be $2 \ell$ measurements from $\bar{u}(\hat{u})$ and $\bar{y}(\hat{u})$, given by (8) and (9), respectively, with sampling period $\delta=\left(u_{\max }-u_{\min }\right) / \ell$. Then, we identify system matrices $\hat{A}_{+}, \hat{A}_{-}$, $\hat{B}_{+}, \hat{B}_{-}$, and $\hat{C}$ to approximately satisfy the PWARX system

$$
\begin{aligned}
x_{k+1} & = \begin{cases}\hat{A}_{+} x_{k}+\hat{B}_{+} u_{k}, & k=0, \ldots, \ell-1 \\
\hat{A}_{-} x_{k}+\hat{B}_{-} u_{k}, & k=1, \ldots, 2 \ell-1\end{cases} \\
y_{k} & =\hat{C} x_{k}
\end{aligned}
$$

where $x_{k} \in \mathbb{R}^{n}, k=0, \ldots, 2 \ell-1$. For the discrete system (10), (11), the convergence condition is $\rho\left(\hat{A}_{+}^{\ell} \hat{A}_{-}^{\ell}\right)<1$. Since the switching time of (10), (11) is fixed, we can use constrained least squares techniques to identify the system matrices. To construct the state vector $x_{k}$ from $u_{k}$ and $y_{k}, k=0, \ldots, 2 \ell-1$, we use the nonminimal state-space 
(NMSS) representation approach given in [6]. Then, $\hat{A}_{+}, \hat{B}_{+}, \hat{A}_{-}$and $\hat{B}_{-}$are determined from

$$
\begin{aligned}
& \min _{\hat{A}_{+}, \hat{A}_{-}, \hat{B}_{+}, \hat{B}_{-}}\left\{\left\|\Phi_{2_{+}}-\left[\hat{A}_{+} \quad \hat{B}_{+}\right]\left[\begin{array}{c}
\Phi_{1_{+}} \\
U_{1_{+}}
\end{array}\right]\right\|_{\mathrm{F}}\right. \\
& \left.\left.+\| \Phi_{2_{-}-\left[\hat{A}_{-}\right.} \hat{B}_{-}\right]\left[\begin{array}{l}
\Phi_{1_{-}} \\
U_{1_{-}}
\end{array}\right] \|_{\mathrm{F}}\right\}
\end{aligned}
$$

subject to

$$
\rho\left(\hat{A}_{+}^{\ell} \hat{A}_{-}^{\ell}\right)<1
$$

where

$$
\begin{aligned}
& \Phi_{1_{+}} \triangleq\left[\begin{array}{lll}
x_{m-1} & \cdots & x_{\ell-1}
\end{array}\right], \quad \Phi_{2_{+}} \triangleq\left[\begin{array}{lll}
x_{m} & \cdots & x_{\ell}
\end{array}\right] \\
& \Phi_{1_{-}} \triangleq\left[\begin{array}{lll}
x_{\ell} & \cdots & x_{2 \ell-2}
\end{array}\right], \quad \Phi_{2_{-}} \triangleq\left[\begin{array}{lll}
x_{\ell+1} & \cdots & x_{2 \ell-1}
\end{array}\right] \\
& U_{1_{+}} \triangleq\left[\begin{array}{lll}
u_{m-1} & \cdots & u_{\ell-1}
\end{array}\right], \quad U_{1_{-}} \triangleq\left[\begin{array}{lll}
u_{\ell} & \cdots & u_{2 \ell-2}
\end{array}\right] .
\end{aligned}
$$

Since the convergence condition (13) is not convex, we use the fact that, if $\bar{\sigma}(A)<1$ (where $\bar{\sigma}$ denotes maximum singular value), then $\rho\left(A^{\ell}\right) \leq \bar{\sigma}\left(A^{\ell}\right) \leq \bar{\sigma}^{\ell}(A)<1$. Hence, if

$$
\bar{\sigma}\left(\hat{A}_{+}\right)<1
$$

and

$$
\bar{\sigma}\left(\hat{A}_{-}\right)<1,
$$

then $\rho\left(\hat{A}_{+}^{\ell} \hat{A}_{-}^{\ell}\right) \leq \bar{\sigma}\left(\hat{A}_{+}^{\ell} \hat{A}_{-}^{\ell}\right) \leq \bar{\sigma}^{\ell}\left(\hat{A}_{+}\right) \bar{\sigma}^{\ell}\left(\hat{A}_{-}\right)<1$ and, thus, (13) is satisfied. The conditions (14) and (15) are conservative relative to (13) but have the advantage of being convex. We thus replace the original constrained least squares problem (12), (13) by the separate constrained least squares problems

$$
\begin{array}{ll}
\min _{\substack{\hat{A}_{+}, \hat{B}_{+} \\
\text {subject to }}} \quad \| \Phi_{2_{+}}-\left[\hat{A}_{+}\right)<1 & \left.\hat{B}_{+}\right]\left[\begin{array}{c}
\Phi_{1_{+}} \\
U_{1_{+}}
\end{array}\right] \|_{\mathrm{F}},
\end{array}
$$

and

$$
\begin{aligned}
& \min _{\hat{A}_{-}, \hat{B}_{-}} \quad\left\|\Phi_{2}-\left[\hat{A}_{-} \hat{B}_{-}\right]\left[\begin{array}{l}
\Phi_{1} \\
U_{1_{-}}
\end{array}\right]\right\|_{\mathrm{F}}, \\
& \text { subject to } \quad \bar{\sigma}\left(\hat{A}_{-}\right)<1 \text {. }
\end{aligned}
$$

Finally, we convert the identified PWARX models by using the bilinear transformation to obtain the continuous-time parameters $A_{+}$, $A_{-}, B_{+}, B_{-}$, and $C$.

To reduce the conservatism inherent in using (14), (15) in place of (13), we allow $\bar{\sigma}\left(A_{+}\right)<q$ and $\bar{\sigma}\left(A_{-}\right)<q$, where $q \geq 1$. The condition $\rho\left(A_{+}^{\ell} A_{-}^{\ell}\right)<1$ is checked a posteriori.

Example 1: Suppose $\hat{y}_{+}(u)$ and $\hat{y}_{-}(u)$ are given as in Fig. 1 from the rate-independent semilinear Duhem model (1), (2) with

$$
\begin{aligned}
A & =g_{+} A, \quad A_{-}=g_{-} A, \quad B=g_{+} B, \quad B=g_{-} B \\
A & =\left[\begin{array}{cc}
-1 & 4 \\
-4 & -1
\end{array}\right], \quad B=\left[\begin{array}{l}
0 \\
1
\end{array}\right], \quad C=\left[\begin{array}{ll}
0 & 1
\end{array}\right], \\
g_{+} & =1, \quad g_{-}=-1, \quad x_{0}=\left[\begin{array}{ll}
0.15 & 0.15
\end{array}\right]^{\mathrm{T}}
\end{aligned}
$$

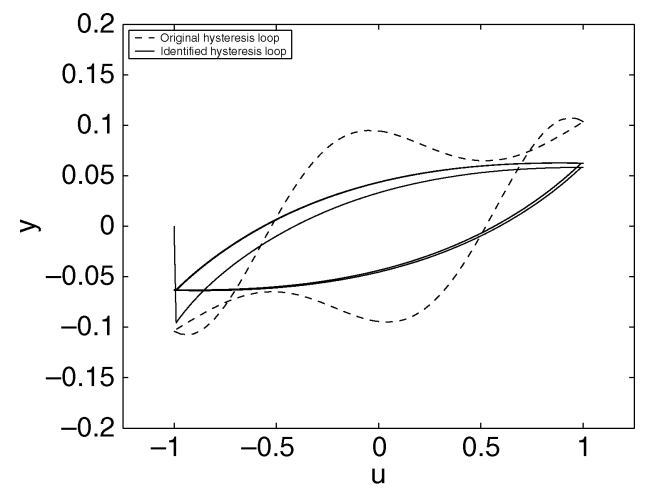

(a)

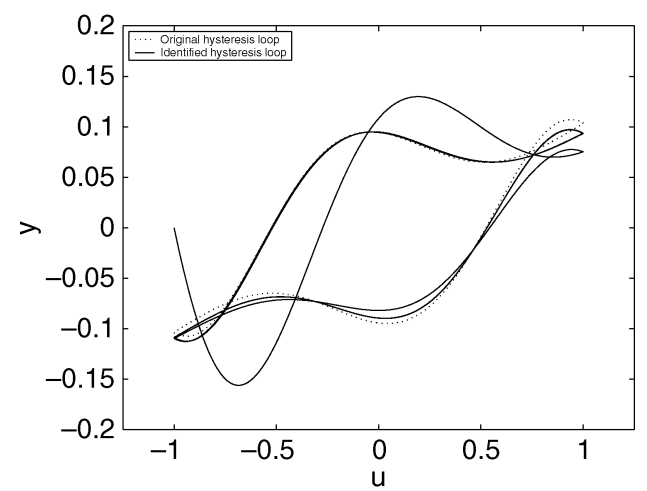

(b)

Fig. 1. Identification of $\hat{y}_{+}(u), \hat{y}_{-}(u)$ of Example 1 with (a) $q=1$ and (b) $q=7$.

and $u(t)=\sin t, t \geq 0$. Identification is performed with $m=2$, and thus the identified system is of order 3. Fig. 1(a) shows the inputoutput map of the identified system with $q=1$. Although $\rho\left(\hat{A}_{+}^{\ell} \hat{A}_{-}^{\ell}\right)=$ 0.0262 and thus the convergence condition is met, the least squares cost (12) is 0.0553 and the input-output map poorly fits $\hat{y}_{+}(u)$ and $\hat{y}_{-}(u)$. The upper bound $q=1$ is now increased to $q=7$, which yields $\rho\left(\hat{A}_{+}^{\ell} \hat{A}_{-}^{\ell}\right)=0.134$. The input-output map of the identified model provides a better fit of the original hysteresis map as shown in Fig. 1(b), and the least squares cost is $5.12 \times 10^{-9}$.

\section{RATE-DePENDENT Semilinear DuHEM Model}

We now consider the SISO rate-dependent semilinear Duhem model

$$
\begin{aligned}
& \dot{x}(t)=(A x(t)+B u(t)) g(\dot{u}(t)) \\
& y(t)=C x(t), \quad x(0)=x_{0}, \quad t \geq 0
\end{aligned}
$$

where $A \in \mathbb{R}^{n \times n}, B \in \mathbb{R}^{n}$, and $C \in \mathbb{R}^{1 \times n}$, and $g: \mathbb{R} \rightarrow \mathbb{R}$ is continuous and satisfies $g(\dot{u})=0$ if and only if $\dot{u}=0$.

Consider the rate-dependent semilinear Duhem model (18), (19), where $u(t)$ is the periodic triangle wave with period $T$ shown as Fig. 2 given by

$$
u(t)= \begin{cases}a t-a q T-\frac{a}{2} T_{a}, & q T \leq t<q T+T_{a} \\ b t-b(q+1) T+\frac{b}{2} T_{b}, & q T+T_{a} \leq t<(q+1) T\end{cases}
$$




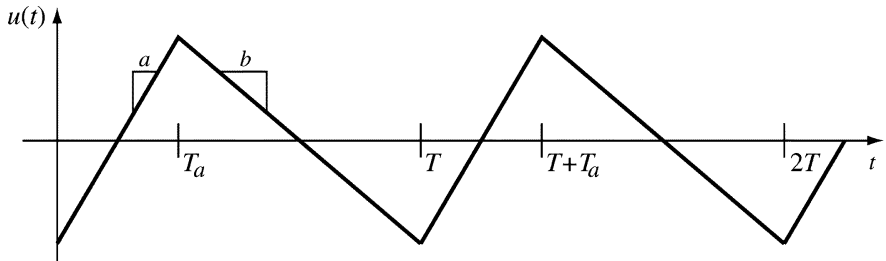

Fig. 2. Triangle wave $u(t)$ defined in (20).

where $a>0, b<0, T_{a}>0, T_{b}>0, T=T_{a}+T_{b}$, and $q=0,1, \ldots$ Then, (18) and (19) become

$$
\begin{aligned}
& \dot{x}(t)= \begin{cases}g(a) A x(t)+g(a) B u(t), & q T \leq t<q T+T_{a} \\
g(b) A x(t)+g(b) B u(t), & q T+T_{a} \leq t<(q+1) T\end{cases} \\
& y(t)=C x(t), \quad x(0)=x_{0}, \quad q=0,1, \ldots
\end{aligned}
$$

Note that (21), (22) can be viewed as a switching linear time-invariant system with switching periods $T_{a}$ and $T_{b}$. The following result will be useful in the next section.

Propsosition 2: Let

$$
\begin{aligned}
& \dot{x}(t)=A x(t)+B u(t), \\
& y(t)=C x(t), \quad x(0)=x_{0}, \quad t \geq 0
\end{aligned}
$$

where $A \in \mathbb{R}^{n \times n}, B \in \mathbb{R}^{n}, C \in \mathbb{R}^{1 \times n}$ are a state-space realization of the linear ordinary differential equation

$$
\begin{array}{r}
y^{(n)}(t)+\alpha_{1} y^{(n-1)}(t)+\cdots+\alpha_{n-1} \dot{y}(t)+\alpha_{n} y(t) \\
=\beta_{1} u^{(n-1)}(t)+\cdots+\beta_{n-1} \dot{u}(t)+\beta_{n} u(t)
\end{array}
$$

for $t \geq 0$, with $\left(y(0), \dot{y}(0), \ldots, y^{(n-1)}(0)\right)=\left(y_{0}, y_{1}, \ldots, y_{n-1}\right)$. Then, for all $\eta \neq 0$

$$
\begin{aligned}
& \dot{x}_{\eta}(t)=\eta A x_{\eta}(t)+\eta B u(t), \\
& y_{\eta}(t)=C x_{\eta}(t), \quad x_{\eta}(0)=x_{\eta_{0}}, \quad t \geq 0
\end{aligned}
$$

is a state-space realization of the linear ordinary differential equation

$$
\begin{gathered}
y_{\eta}^{(n)}(t)+\eta \alpha_{1} y_{\eta}^{(n-1)}(t)+\cdots+\eta^{n-1} \alpha_{n-1} \dot{y}_{\eta}(t)+\eta^{n} \alpha_{n} y_{\eta}(t) \\
=\eta \beta_{1} u^{(n-1)}(t)+\cdots+\eta^{n-1} \beta_{n-1} \dot{u}(t)+\eta^{n} \beta_{n} u(t)
\end{gathered}
$$

for $t \geq 0, \quad$ with $\left(y_{\eta}(0), \dot{y}_{\eta}(0), \ldots, y_{\eta}^{(n-1)}(0)\right)=$ $\left(y_{\eta_{0}}, y_{\eta_{1}}, \ldots, y_{\eta_{n-1}}\right)$.

Proof: Without loss of generality, let $A, B$, and $C$ be in observable canonical form

$$
A=\left[\begin{array}{cccc}
-\alpha_{1} & 1 & \cdots & 0 \\
\vdots & \vdots & \ddots & \vdots \\
-\alpha_{n-1} & 0 & \cdots & 1 \\
-\alpha_{n} & 0 & \cdots & 0
\end{array}\right], \quad B=\left[\begin{array}{c}
\beta_{1} \\
\vdots \\
\beta_{n-1} \\
\beta_{n}
\end{array}\right], \quad C=\left[\begin{array}{c}
1 \\
0 \\
\vdots \\
0
\end{array}\right]^{\mathrm{T}}
$$

Next, define $T \triangleq \operatorname{diag}\left(1, \eta^{-1}, \ldots, \eta^{-n+1}\right)$ and note that

$$
\begin{aligned}
\eta T^{-1} A T & =\left[\begin{array}{cccc}
-\eta \alpha_{1} & 1 & \cdots & 0 \\
\vdots & \vdots & \ddots & \vdots \\
-\eta^{n-1} \alpha_{n-1} & 0 & \cdots & 1 \\
-\eta^{n} \alpha_{n} & 0 & \cdots & 0
\end{array}\right] \\
\eta T^{-1} B & =\left[\begin{array}{c}
\eta \beta_{1} \\
\vdots \\
\eta^{n-1} \beta_{n-1} \\
\eta^{n} \beta_{n}
\end{array}\right] \\
C T & =\left[\begin{array}{lll}
1 & 0 & \ldots
\end{array}\right]
\end{aligned}
$$

which is a realization of (28).

\section{IDENTIFICATION OF THE RATE-DEPENDENT SEMILINEAR DUHEM MODEL}

Consider the rate-dependent semilinear Duhem model (18), (19), where $u(t), t \geq 0$, is the periodic triangle wave with period $T$ given by (20). Suppose that there exists a periodic solution $x(t)$ of (18), and let $y(t), t \geq 0$, be given by (19). Then, it follows from (21) and (22) that (18) and (19) can be written as

$$
\dot{x}(t)=g(a) A x(t)+g(a) B u(t), \quad y(t)=C x(t)
$$

for $0 \leq t<T_{a}$, and

$$
\dot{x}(t)=g(b) A x(t)+g(b) B u(t), \quad y(t)=C x(t)
$$

for $T_{a} \leq t<T$. Since (29), (30) is a switching linear time-invariant system, we can identify $g(a) A, g(a) B$ on $\left[0, T_{a}\right)$, and $g(b) A, g(b) B$ on $\left[T_{a}, T\right)$ using the standard least squares identification method, where $u(t)$ is monotonically increasing and monotonically decreasing, respectively. Let $u_{k}$ and $y_{k}, k=0,1, \ldots, \ell-1$, be measurements of $u(t)$ and $y(t)$, respectively, where $t \in[0, T)$, with a sampling period $\delta=T / \ell$. Let $\ell_{+}>0, \ell_{-}>0$, and $\ell=\ell_{+}+\ell_{-}$, where $u_{k}$ is monotonically increasing for $k=0, \ldots, \ell_{+}-1$ and monotonically decreasing for $k=\ell_{+}, \ldots \ell-1$.

Next, suppose that $u_{k}$ and $y_{k}$ satisfy the $n$-dimensional DARMA model

$$
y_{k+1}=-\hat{\alpha}_{1}^{+} y_{k}-\cdots-\hat{\alpha}_{n}^{+} y_{k-n+1}+\hat{\beta}_{1}^{+} u_{k}+\cdots+\hat{\beta}_{n}^{+} u_{k-n+1}
$$

for $k=0,1, \ldots, \ell_{+}-1$, and

$$
y_{k+1}=-\hat{\alpha}_{1}^{-} y_{k}-\cdots-\hat{\alpha}_{n}^{-} y_{k-n+1}+\hat{\beta}_{1}^{-} u_{k}+\cdots+\hat{\beta}_{n}^{-} u_{k-n+1}
$$

for $k=\ell_{+}, \ell_{+}+1, \ldots, \ell-1$, where the model order $n \leq(\ell-2) / 2$ is selected empirically, and $\hat{\alpha}_{j}^{+}, \hat{\alpha}_{j}^{-}, \hat{\beta}_{j}^{+}$, and $\hat{\beta}_{j}^{-}, j=1, \ldots, n$, are system parameters. Now, by defining

$$
\begin{gathered}
Y_{+} \triangleq\left[\begin{array}{lll}
y_{n} & \cdots & y_{\ell_{+}-1}
\end{array}\right]^{\mathrm{T}}, \quad Y_{-} \triangleq\left[\begin{array}{llll}
y_{\ell_{+}+n} & \cdots & y_{\ell-1}
\end{array}\right]^{\mathrm{T}} \\
\Phi_{+} \triangleq\left[\begin{array}{cccccc}
-y_{n-1} & \cdots & -y_{0} & u_{n-1} & \cdots & u_{0} \\
\vdots & \ddots & \vdots & \vdots & \ddots & \vdots \\
-y_{\ell_{+}-2} & \cdots & -y_{\ell_{+}-n-1} & u_{\ell_{+}-2} & \cdots & u_{\ell_{+}-n-1}
\end{array}\right] \\
\Phi_{-} \triangleq\left[\begin{array}{cccccc}
-y_{\ell_{+}+n-1} & \cdots & -y_{\ell_{+}} & u_{\ell_{+}+n-1} & \cdots & u_{\ell_{+}} \\
\vdots & \ddots & \vdots & \vdots & \ddots & \vdots \\
-y_{\ell-2} & \cdots & -y_{\ell-n-1} & u_{\ell-2} & \cdots & u_{\ell-n-1}
\end{array}\right]
\end{gathered}
$$


(31) and (32) can be rewritten as

$$
Y_{+}=\Phi_{+} \hat{\theta}_{+}, \quad Y_{-}=\Phi_{-} \hat{\theta}_{-}
$$

where $\hat{\theta}_{+} \triangleq\left[\hat{\alpha}_{1}^{+} \cdots \hat{\alpha}_{n}^{+} \hat{\beta}_{1}^{+} \cdots \hat{\beta}_{n}^{+}\right]^{\mathrm{T}}, \hat{\theta}_{-} \triangleq\left[\hat{\alpha}_{1}^{-} \cdots \hat{\alpha}_{n}^{-} \hat{\beta}_{1}^{-} \cdots \hat{\beta}_{n}^{-}\right]^{\mathrm{T}}$, and least-squares estimates of the system parameters are given by

$$
\hat{\theta}_{+}=\Phi_{+}^{\dagger} Y_{+}, \quad \hat{\theta}_{-}=\Phi_{-}^{\dagger} Y_{-}
$$

where ()$^{\dagger}$ denotes the Moore-Penrose generalized inverse.

Next, to obtain coefficients for the continuous-time system, we convert the DARMA models (31) and (32) using the bilinear transformation into the continuous-time linear ordinary differential equations

$$
\begin{aligned}
y^{(n)}(t)+\alpha_{1}^{+} y^{(n-1)}(t)+\cdots+\alpha_{n-1}^{+} \dot{y}(t)+\alpha_{n}^{+} y(t) \\
=\beta_{1}^{+} u^{(n-1)}(t)+\cdots+\beta_{n-1}^{+} \dot{u}(t)+\beta_{n}^{+} u(t)
\end{aligned}
$$

for $0 \leq t<T_{a}$, and

$$
\begin{aligned}
y^{(n)}(t)+\alpha_{1}^{-} y^{(n-1)}(t)+\cdots+\alpha_{n-1}^{-} \dot{y}(t)+\alpha_{n}^{-} y(t) \\
=\beta_{1}^{-} u^{(n-1)}(t)+\cdots+\beta_{n-1}^{-} \dot{u}(t)+\beta_{n}^{-} u(t)
\end{aligned}
$$

for $T_{a} \leq t<T$, respectively, where $\alpha_{j}^{+}, \alpha_{j}^{-}, \beta_{j}^{+}$, and $\beta_{j}^{-}, j=$ $1, \ldots, n$, are system parameters. Now, consider

$$
\begin{aligned}
\dot{x}(t) & =A x(t)+B u(t), \\
y(t) & =C x(t), \quad x(0)=x_{0}, \quad t \geq 0
\end{aligned}
$$

and let (37), (38) be a state-space realization of the linear ordinary differential equation

$$
\begin{aligned}
y^{(n)}(t)+\alpha_{1} y^{(n-1)}(t)+\cdots+\alpha_{n-1} \dot{y}(t)+\alpha_{n} y(t) \\
=\beta_{1} u^{(n-1)}(t)+\cdots+\beta_{n-1} \dot{u}(t)+\beta_{n} u(t)
\end{aligned}
$$

for $t \geq 0$, with $\left(y(0), \dot{y}(0), \ldots, y^{(n-1)}(0)\right)=\left(y_{0}, y_{1}, \ldots, y_{n-1}\right)$. Then, Proposition 2 implies that (35) and (36) are equivalent, respectively, to (28) with $\eta=g(a)$ and (28) with $\eta=g(b)$. Comparing the coefficients of (35) with those of (28) with $\eta=g(a)$ yields

$$
\begin{aligned}
& \alpha_{1}^{+}=g(a) \alpha_{1}, \alpha_{2}^{+}=g^{2}(a) \alpha_{2}, \ldots, \alpha_{n}^{+}=g^{n}(a) \alpha_{n} \\
& \beta_{1}^{+}=g(a) \beta_{1}, \beta_{2}^{+}=g^{2}(a) \beta_{2}, \ldots, \beta_{n}^{+}=g^{n}(a) \beta_{n}
\end{aligned}
$$

while comparing the coefficients of (36) with those of (28) with $\eta=$ $g(b)$ yields

$$
\begin{aligned}
& \alpha_{1}^{-}=g(b) \alpha_{1}, \alpha_{2}^{-}=g^{2}(b) \alpha_{2}, \ldots, \alpha_{n}^{-}=g^{n}(b) \alpha_{n} \\
& \beta_{1}^{-}=g(b) \beta_{1}, \beta_{2}^{-}=g^{2}(b) \beta_{2}, \ldots, \beta_{n}^{-}=g^{n}(b) \beta_{n} .
\end{aligned}
$$

Hence, $g(a)$ and $g(b)$ are given by

$$
g(a)=\frac{\alpha_{1}^{+}}{\alpha_{1}}=\frac{\beta_{1}^{+}}{\beta_{1}}, \quad g(b)=\frac{\alpha_{1}^{-}}{\alpha_{1}}=\frac{\beta_{1}^{-}}{\beta_{1}} .
$$

However, (44) provides only an approximation of $g(a)$ and $g(b)$ due to the bilinear transformation and the presence of noise. Thus, we use estimates $\hat{g}(a)$ of $g(a)$ and $\hat{g}(b)$ of $g(b)$ given by the mean values

$$
\hat{g}(a)=\frac{\alpha_{1}^{+} \beta_{1}+\alpha_{1} \beta_{1}^{+}}{2 \alpha_{1} \beta_{1}}, \quad \hat{g}(b)=\frac{\alpha_{1}^{-} \beta_{1}+\alpha_{1} \beta_{1}^{-}}{2 \alpha_{1} \beta_{1}} .
$$

The estimates $\hat{A}, \hat{B}$, and $\hat{C}$ of the system matrices can be determined from the coefficients $\alpha_{j}^{+}$and $\beta_{j}^{+}, j=1, \ldots, n$, of (35). Specifically, letting $g(a)=1$, it follows from (40), (41) that

$$
\alpha_{j}=\alpha_{j}^{+} \quad \beta_{j}=\beta_{j}^{+}
$$

for $j=1, \ldots, n$, and $\hat{A}, \hat{B}$, and $\hat{C}$ are given by the observable canonical form. Note that, without loss of generality, we can set $g(a)=1$ since (29), (30) can be written as

$$
\begin{aligned}
& \dot{x}(t)= \begin{cases}\tilde{A} x(t)+\tilde{B} u(t), & 0 \leq t<T_{a} \\
\frac{g(b)}{g(a)} \tilde{A} x(t)+\frac{g(b)}{g(a)} \tilde{B} u(t), & T_{a} \leq t<T\end{cases} \\
& y(t)=C x(t),
\end{aligned}
$$

where $\tilde{A} \triangleq(1 / g(a)) A$ and $\tilde{B} \triangleq(1 / g(a)) A$ and where, by assumption, $g(\dot{u})=0$ if and only if $\dot{u}=0$. Then, $\hat{g}(b)$ is determined by (45).

Once $\hat{A}, \hat{B}, \hat{C}$, and $\hat{g}(b)$ are determined, we identify $g(\dot{u})$ pointwise using (45) by applying the identification procedure with triangle waves having different slopes. Specifically, let $p \geq 2$ be an integer and, for $i=1, \ldots, p$, let $u_{i}(t), t \geq 0$, be a triangle wave input as defined in (20) with switching periods $T_{a_{i}}$ and $T_{b_{i}}$, where $T_{a_{1}}<T_{a_{2}}<\cdots<$ $T_{a_{p}}$ and $T_{b_{1}}<T_{b_{2}}<\cdots<T_{b_{p}}$, and slopes $a_{i}>0$ and $b_{i}<$ 0 . Let $y_{i}(t), t \geq 0$, be the corresponding steady-state output of the rate-dependent semilinear Duhem model (18), (19), which is periodic with period $T_{i}=T_{a_{i}}+T_{b_{i}}$ so that the input-output map of $u_{i}(t)$ and $y_{i}(t)$ forms a closed curve. Now, for $i=1, \ldots, p$, identification of the rate-dependent semilinear Duhem model consists of the following steps.

1) Using $\ell_{i}$ measurements of $u_{i}(t)$ and $y_{i}(t)$ with fixed sampling time $\delta=T / \ell$, determine the coefficients $\hat{\alpha}_{i, j}^{+}, \hat{\beta}_{i, j}^{+}, \hat{\alpha}_{i, j}^{+}$, and $\hat{\beta}_{i, j}^{+}$, $j=1, \ldots, n$, of the DARMA models (31), (32) with $\hat{\alpha}_{j}^{+}=\hat{\alpha}_{i, j}^{+}$, $\hat{\beta}_{j}^{+}=\hat{\beta}_{i, j}^{+}, \hat{\alpha}_{j}^{-}=\hat{\alpha}_{i, j}^{-}$and $\hat{\beta}_{j}^{-}=\hat{\beta}_{i, j}^{-}, j=1, \ldots, n$.

2) Determine the coefficients $\alpha_{i, j}^{+}, \beta_{i, j}^{+}, \alpha_{i, j}^{+}$and $\beta_{i, j}^{+}, j=1, \ldots, n$, of the linear ordinary differential equations (35), (36) with $\alpha_{j}^{+}=$ $\alpha_{i, j}^{+}, \beta_{j}^{+}=\beta_{i, j}^{+}, \alpha_{j}^{-}=\alpha_{i, j}^{-}$, and $\beta_{j}^{-}=\beta_{i, j}^{-}, j=1, \ldots, n$, by converting the DARMA models from Step 1) to the linear differential equations through the bilinear transformation.

3) If $i=1$, estimate the system matrices $\hat{A}, \hat{B}$, and $\hat{C}$ by

$$
\begin{aligned}
& \hat{A}=\left[\begin{array}{cccc}
\alpha_{1,1}^{+} & 1 & \cdots & 0 \\
\vdots & \vdots & \ddots & \vdots \\
-\alpha_{n, 1}^{+} & 0 & \cdots & 1
\end{array}\right], \quad \hat{B}=\left[\begin{array}{c}
\beta_{1,1}^{+} \\
\vdots \\
\beta_{n, 1}^{+}
\end{array}\right], \\
& \hat{C}=\left[\begin{array}{llll}
1 & 0 & \cdots & 0
\end{array}\right] .
\end{aligned}
$$

Then, set $\hat{g}\left(a_{1}\right)=1$, and determine $\hat{g}\left(b_{1}\right)$ from (45) with $\alpha_{1}=$ $\alpha_{1,1}^{+}, \beta_{1}=\beta_{1,1}^{+}, \alpha_{1}^{-}=\alpha_{1,1}^{-}$, and $\beta_{1}^{-}=\beta_{1,1}^{-}$.

4) If $i>1$, determine $\hat{g}\left(a_{i}\right)$ and $\hat{g}\left(b_{i}\right)$ by

$$
\begin{aligned}
\hat{g}\left(a_{i}\right) & =\frac{\alpha_{i, 1}^{+} \beta_{1}^{+}+\alpha_{1}^{+} \beta_{i, 1}^{+}}{2 \alpha_{1}^{+} \beta_{1}^{+}} \\
\hat{g}\left(b_{i}\right) & =\frac{\alpha_{i, 1}^{-} \beta_{1}^{+}+\alpha_{1}^{+} \beta_{i, 1}^{-}}{2 \alpha_{1}^{+} \beta_{1}^{+}} .
\end{aligned}
$$




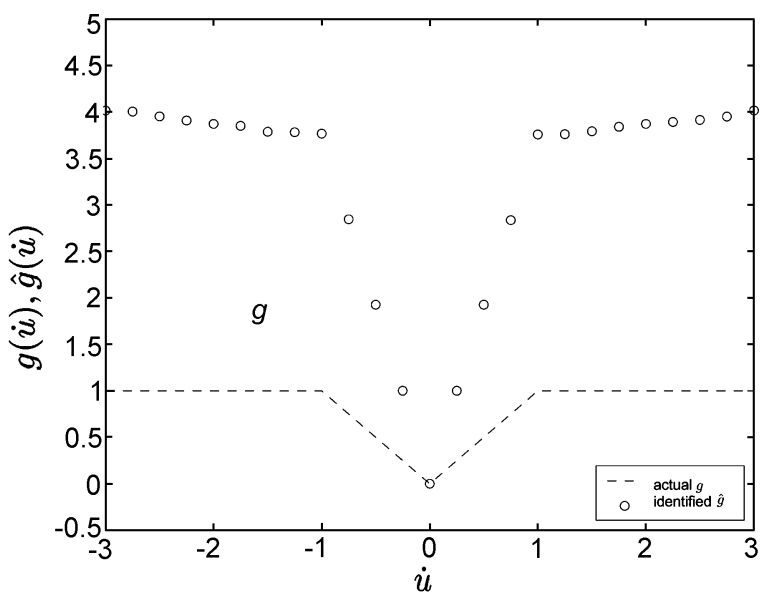

(a)

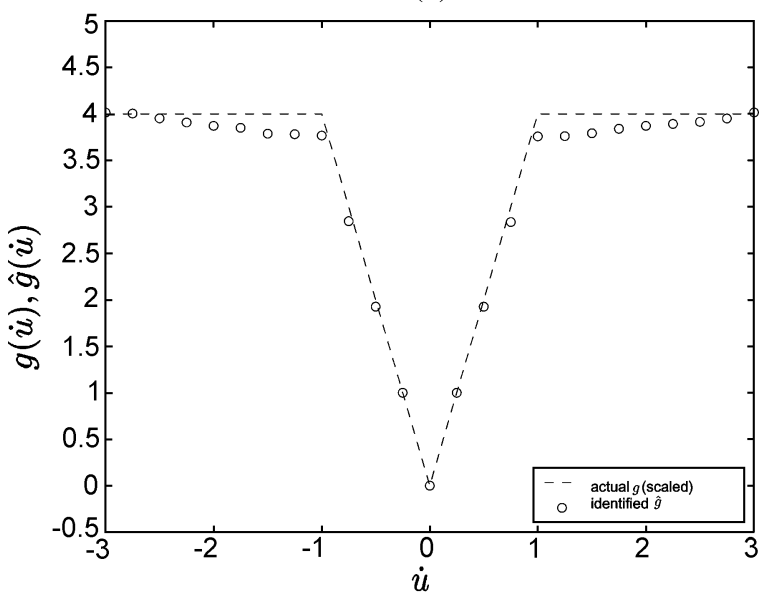

(b)

Fig. 3. (a) Original and (b) scaled $g$ given by (52) (dashed) and the pointwise identification $\hat{g}$ (circles) of Example 2.

Example 2: Consider the rate-dependent semilinear Duhem model (18), (19) with

$$
A=-1, \quad B=1, \quad C=1,
$$

and $g$ given by

$$
g(v)= \begin{cases}|v|, & |v| \leq 1 \\ 1, & |v|>1\end{cases}
$$

Note that $g$ is not positively homogeneous. The identification method developed in Section VI is used with 12 triangle waves, where $a_{i}=$ $0.25,0.5,0.75,1,1.25,1.5,1.75,2,2.25,2.5,2.75,3, b_{i}=-a_{i}$, and $T_{a_{i}}=T_{b_{i}}=4 / a_{i}, i=1, \ldots, 12$. For $i=1, \ldots, 12$, the input $u_{i}$ and the output $y_{i}$ are sampled with $\delta=0.13$. We corrupt the output with uniformly distributed measurement noise whose peak-to-peak amplitude is 0.1 . The system parameters are identified as

$$
\hat{A}=-0.2501, \quad \hat{B}=0.25, \quad \hat{C}=1,
$$

and $g(\dot{u})$ is identified in pointwise fashion as shown in Fig. 3(a). Note that the system parameters (53) and the identified $\hat{g}$ are scaled by 0.25 and 4, respectively, since $g\left(a_{1}\right)=g(0.25)=0.25$. Fig. 3(b) shows the scaled graph, which shows that the identified $\hat{g}(\dot{u})$ closely fits
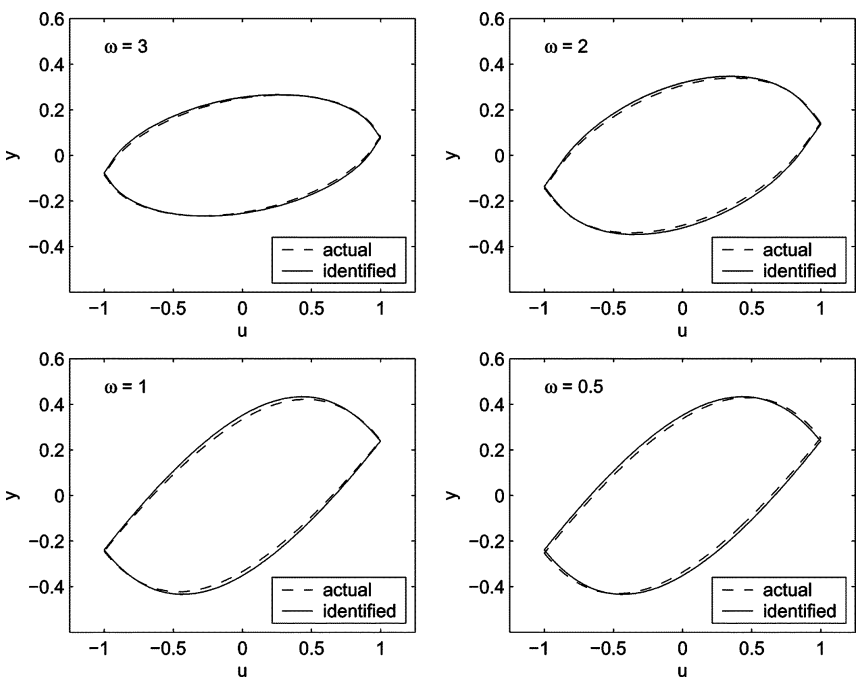

Fig. 4. Input-output maps of original (dashed) and identified (solid) rate-dependent semilinear Duhem model of Example 2 under $u(t)=\sin \omega t$.

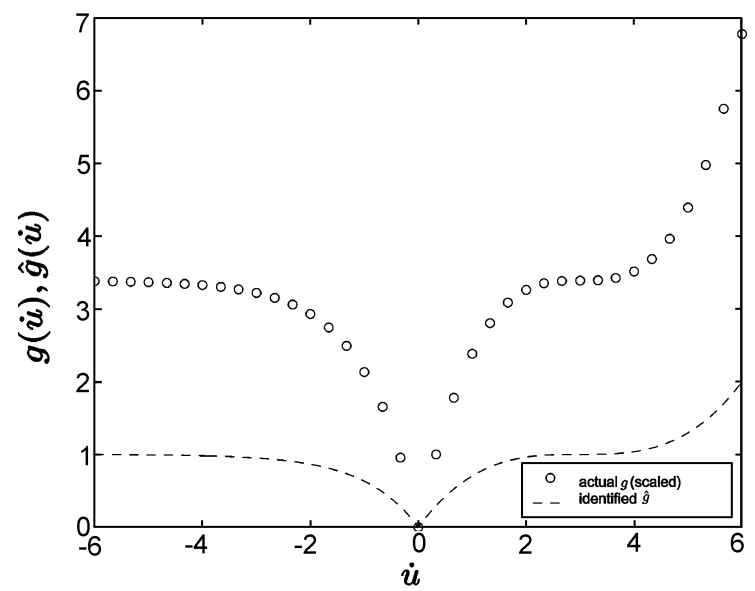

(a)

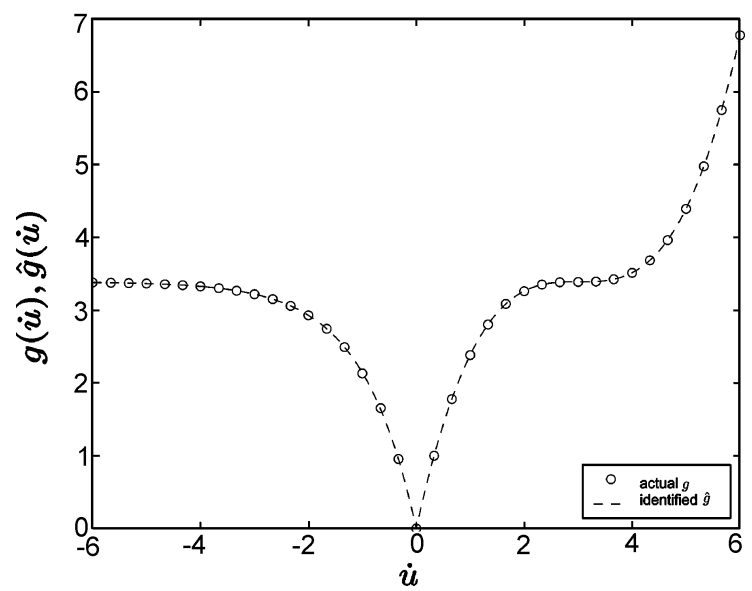

(b)

Fig. 5. (a) Original and (b) scaled $g$ given by (55) (dashed) and the pointwiseidentified $\hat{g}$ (circles) of Example 3.

the actual $g$. Fig. 4 shows the input-output maps of the actual and identified rate-dependent semilinear Duhem model with the identified $\hat{g}$ under sinusoidal inputs. 

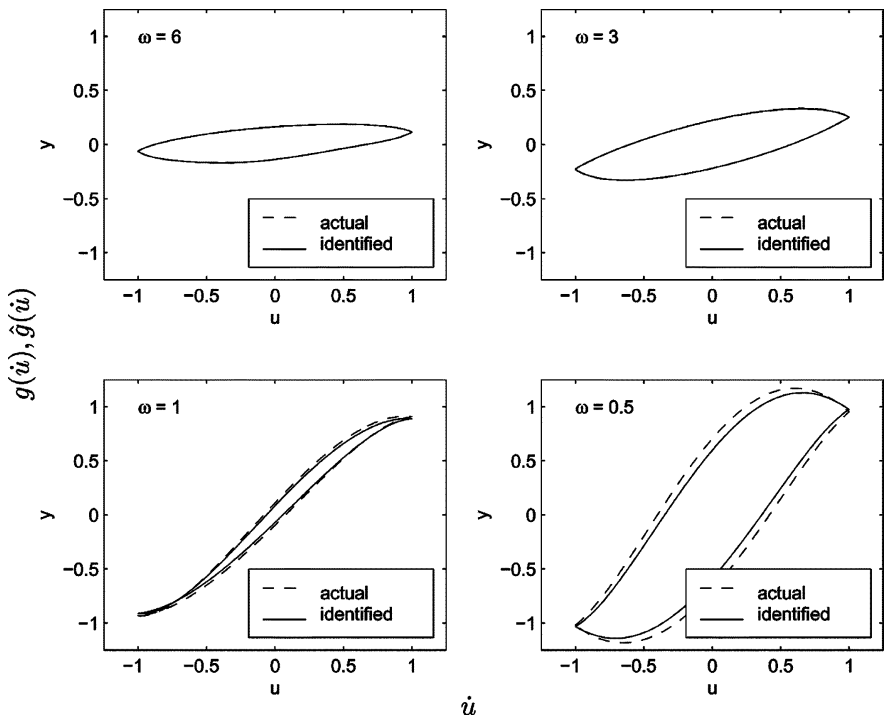

Fig. 6. Input-output maps of actual (dashed) and identified rate-dependent semilinear Duhem model of Example 3 under $u(t)=\sin \omega t$ without measurement noise.

Example 3: Consider the rate-dependent semilinear Duhem model (18), (19) with

$$
A=\left[\begin{array}{cc}
0 & 1 \\
-3 & -1
\end{array}\right], \quad B=\left[\begin{array}{l}
1 \\
0
\end{array}\right], \quad C=\left[\begin{array}{ll}
1 & 1
\end{array}\right]
$$

and $g$ given by

$$
g(v)= \begin{cases}\left(\frac{v}{3}-1\right)^{3}+1, & v \geq 0 \\ 1-e^{v}, & v<0\end{cases}
$$

For identification, 14 triangle waves are used with $a_{i}=0.25,0.5,0.75,1,1.5,2,2.5,3,3.5,4,4.5,5,5.5,6$, $b_{i}=-a_{i}$, and $T_{a_{i}}=T_{b_{i}}=4 / a_{i}, i=1, \ldots, 14$. Fig. 6 shows the input-output maps of the actual and identified rate-dependent semilinear Duhem model with the identified $\hat{g}$ under sinusoid inputs without measurement noise. The sensitivity of the identified parameters can be estimated by standard least squares techniques.

\section{CONCLUSION}

In this note, we developed identification methods for rate-independent and rate-dependent semilinear Duhem models. For the rate-independent model, the nonlinear identification was facilitated by reparameterization in terms of the input, resulting in a linear switching system. The rate-dependent model was analyzed as a linear switching system under triangle wave inputs. Least squares methods were developed to identify the system parameters, and the scalar function of the input derivative for the rate-dependent semilinear Duhem model was identified in pointwise fashion.

\section{REFERENCES}

[1] J. Oh and D. S. Bernstein, "Semilinear Duhem model for rate-independent and rate-dependent hysteresis," IEEE Trans. Autom. Control, vol. 50, no. 5, pp. 631-645, May 2005.

[2] L. O. Chua and S. C. Bass, "A generalized hysteresis model," IEEE Trans. Circuit Theory, vol. CT-19, no. 1, pp. 36-48, Jan. 1972.

[3] B. D. Coleman and M. L. Hodgdon, "A constitutive relation for rateindependent hysteresis in ferromagnetically soft materials," Int. J. Eng. Sci., vol. 24, no. 6, pp. 897-919, 1986.

[4] J. W. Macki, P. Nistri, and P. Zecca, "Mathematical models for hysteresis," SIAM Rev., vol. 35, no. 1, pp. 94-123, 1993.

[5] J. Oh, A. K. Padthe, D. S. Bernstein, D. D. Rizos, and S. D. Fassois, "Duhem models for hysteresis in sliding and presliding friction," in Proc. Amer. Control Conf., Seville, Spain, 2005, pp. 8132-8137.

[6] C. J. Yaylor, A. Chotai, and P. C. Young, "State space control system design based on non-minimal state-variable feedback: further generalization and unification results," Int. J. Control, vol. 73, no. 14, pp. 1329-1345, 2000. 